\section{Tendencia en la sobrevida de pacientes hospitalizados por infarto agudo de miocardio según nivel socioeconómico: Chile, 2002-2012}

\author{
CAROLINA NAZZAL ${ }^{1, \mathrm{a}, \mathrm{d}, \mathrm{e}}$, FAUSTINO ALONSO ${ }^{1}$, \\ FRANCISCO CERECERA ${ }^{1, b, d}$, JOSÉ MIGUEL OJEDA ${ }^{1, c}$
}

\section{Association between socioeconomic status and survival after a first episode of myocardial infarction}

Background: A low socioeconomic status is associated with higher overall mortality rates. Aim: To assess the effect of socioeconomic inequalities on survival of patients hospitalized with a first myocardial infarction. Material and Methods: Analysis of hospital discharge and mortality databases of the Ministry of Health. Patients aged over 15 years discharged between 2002 and 2011 with a first myocardial infarction (code I-21, ICD-10) were identified. Their survival was verified with the mortality registry. Survival from 0 to 28 and from 29 to 365 days was analyzed. Socioeconomic status was determined using the type of health insurance, stratified as public insurance (low and medium status) and private insurance (high status). Prais-Winsten trend $(P-W)$ and Cox survival analyses were done. Results: We analyzed 59,557 patients (69\% males). Sixty three percent were of low socioeconomic status, 19\% medium and 18\% high. Between 2002 and 2011 the increase in survival was higher among patients of low socioeconomic status, mainly in women ( $P$-W coefficients $0.58: 0.31-0.86$ in men and 1.12:0.84-1.41 in women for 0-28 days survival and 0.24:0.09-0.39 in men and 0.48:0.37-0.60 in women for 29-365 days survival, respectively). However, age and year of hospitalization adjusted analysis showed a higher mortality risk among patients of low socioeconomic status at 0-28 days ( HR 1.67:1.53-1.83 for men and 1.49:1.34-1.66 for women) and at 29-365 days (HR 2.30: 1.75-2.71 for men and 1.90:1.56-1.85 for women). Conclusions: Survival after a myocardial infarction improved in the last decade especially in patients of low socioeconomic status. However, subjects of this stratum continue to have a higher mortality.

(Rev Med Chile 2017; 145: 827-836)

Key words: Chile; Myocardial Infarction; Social Class; Survival Rate.
Escuela de Salud Pública, Facultad de Medicina, Universidad de Chile. Santiago, Chile. aEnfermera Matrona. bIngeniero comercial. 'Estudiante Medicina. ${ }^{\mathrm{d}} \mathrm{MPH}$.

ePhD.

Financiamiento: Fondo concursable para el apoyo a la investigación, Insituto de Salud Poblacional - Escuela de Salud Pública, Facultad de Medicina, Universidad de Chile. Los autores no declaran conflicto de intereses.

Recibido el 8 de mayo de 2017 , aceptado el 3 de agosto de 2017

Correspondencia a:

Carolina Nazzal Nazal

Independencia 939. Santiago,

Chile.

cnazzal@med.uchile.cl
$\mathrm{D}$ urante las últimas décadas ha disminuido la mortalidad por enfermedad coronaria, asociado al desarrollo de nuevas estrategias terapéuticas ${ }^{1,2}$. Chile ha presentado igual tendencia: la tasa de mortalidad por enfermedad coronaria se redujo $24 \%$ entre 1998 y $2007^{3}$ y la letalidad por infarto agudo de miocardio (IAM) también ha disminuido ${ }^{4}$. No obstante, se observan inequidades en salud en estos pacientes que desfavorecen a los más pobres ${ }^{3,5-9}$.

Muchos son los factores que determinan el mayor riesgo de enfermar y morir por enfermedad 
coronaria en personas de bajo nivel socioeconómico ${ }^{7,9-11}$. Hay desigualdad en el acceso a tratamientos, tanto en la fase aguda como alejada ${ }^{12-14}$, como también mayor prevalencia de factores de riesgo ${ }^{15,16}$. En un contexto más amplio, también las condiciones estructurales -urbanización de grandes ciudades, calidad del barrio, tráfico o polución ambiental- aumentan la incidencia de eventos coronarios y determinan un peor pronóstico en estos pacientes ${ }^{7,17-20}$.

El abordaje de las inequidades en salud demanda la implementación de políticas públicas a nivel poblacional y de estrategias dirigidas a los individuos de alto riesgo ${ }^{21-23}$. Con este objetivo se implementó progresivamente en Chile, durante la década pasada, un sistema de garantías universales (GES o AUGE) para patologías consideradas prioritarias; con ello se buscó asegurar el acceso y la oportunidad de atención médica a toda la población ${ }^{24}$. Desde su inicio, en 2005, el IAM, la hipertensión arterial y la diabetes fueron incorporados al GES, que incluye la gratuidad para la población de menor ingreso ${ }^{25}$. Paralelamente, se avanzó en políticas públicas para el control del tabaquismo y el sobrepeso ${ }^{3,24}$.

El objetivo de este estudio es evaluar potenciales inequidades socioeconómicas en la sobrevida de pacientes con IAM, empleando como medida de posición socioeconómica el seguro de salud, que refleja el nivel de ingresos y condiciones laborales particulares. Para ello, se analizará la tendencia temporal en la sobrevida de pacientes con IAM en un período de 10 años, entregando información sobre el efecto de las políticas implementadas durante el período, con énfasis en los grupos de diferente nivel socioeconómico.

\section{Materiales y Métodos}

\section{Diseño y bases de datos}

Estudio longitudinal de base poblacional en pacientes mayores de 15 años hospitalizados por un primer IAM durante los años 2002 y 2011 en Chile. Los casos se seleccionaron de las bases de egresos hospitalarios de los años 2002 a 2011, que incluyen todos los hospitales públicos y privados del país. La información sobre la muerte se obtuvo de las bases nacionales de mortalidad de los años 2002 a 2012. Ambas bases se generan en el Departamento de Estadísticas e Información en Salud
(DEIS) y cubren 100\% de las hospitalizaciones y muertes ocurridas en Chile ${ }^{7}$. Las hospitalizaciones por IAM fueron seleccionadas por el código I21 de la Décima versión de la Clasificación Internacional de Enfermedades (CIE-10) en el diagnóstico de egreso. Con el número de identificación único (RUT) se identificaron pacientes con más de un egreso en el período estudiado (IAM recurrente). De los eventos registrados, $28,3 \%$ fueron IAM recurrentes, en cuyo caso se mantuvo sólo el primer egreso.

Para conocer la ocurrencia de muerte se cruzaron ambas bases mediante el RUT; se seleccionaron las muertes de origen cardiovascular con los códigos I00-I99 de la CIE-10. Se eliminaron los casos que tenían información inválida del RUT en la base de egresos hospitalarios. La distribución de estos casos fue: $21 \%$ en $2002,13 \%$ en 2003 , $11 \%$ en $2004,10 \%$ en $2005,11 \%$ en $2006,10 \%$ en $2007,5 \%$ en $2008,5 \%$ en $2009,4 \%$ en 2010 , y $3 \%$ en 2011 ( $8,8 \%$ de la muestra). El estudio fue aprobado por el comité de ética de la Facultad de Medicina de la Universidad de Chile.

\section{Proceso de validación de datos y variables}

Se identificaron los valores perdidos y aberrantes con gráficos de distribución y según criterio de valores cuya diferencia con los percentiles 25 o 75 fuese mayor a 1,5 veces el valor del rango intercuartílico.

También se identificaron las duplicaciones respecto al RUT.

La base de egresos contiene información sobre: tipo de seguro de salud (público, privado, otro), servicio de salud, comuna, región del establecimiento de salud, tipo de beneficiario según previsión de salud (nivel FONASA), fecha de egreso, días de estadía, diagnóstico de egreso, edad, sexo y condición de egreso (vivo o muerto). Se validó el dato "condición de egreso" con el diagnóstico de defunción en la base de mortalidad. Respecto a la información faltante para sexo, en el caso de pacientes con 1 o 2 ingresos se consultó al DEIS; en caso de más de 2 egresos se mantuvo el de mayor referencia. Respecto al seguro de salud se mantuvo la información del primer egreso. Para aquellos que no tenían información en el primer egreso, se consideró el egreso más cercano a la primera hospitalización. Finalmente, se eliminaron de la base aquellos casos que no tenían información de sexo, previsión, diagnóstico y fecha de egreso, 
y se evaluó que esta pérdida de información no afectara el patrón de los datos, comparando estas características entre los casos excluidos con los mantenidos en el análisis.

\section{Nivel socioeconómico (NSE)}

La información del seguro de salud se utilizó para caracterizar el NSE. El seguro público (FONASA) se subdivide en cuatro tramos de acuerdo al ingreso mensual del afiliado, -definido como la suma de todos los ingresos formales que recibe cada mes-. A los tramos más bajos, FONASA A y $B$, pertenecen quienes no tienen ingreso o cuando no supera el ingreso mínimo mensual (IMM). Para los tramos superiores, Cy D, estos exceden el $\mathrm{IMM}^{26}$. El seguro privado incluye los beneficiarios de Instituciones de Salud Previsional (ISAPREs) y seguro de Fuerzas Armadas y otros seguros privados, cuyos cotizantes tienen ingresos similares a los pertenecientes a ISAPREs. De acuerdo con la Encuesta Nacional de Caracterización Socioeconómica 2012 (CASEN), 80,1\% de la población chilena pertenece al seguro público, $13,2 \%$ a privados (ISAPRES), 3,8\% otros y 3,0\% desconocido ${ }^{27}$. Los pacientes fueron agrupados en tres categorías de NSE: bajo (incluye FONASA A/B), medio (FONASA C/D) y alto (pacientes ISAPREs u otro seguro de salud).

\section{Población del estudio}

Durante el período se identificaron 72.108 hospitalizaciones por IAM en 60.651 pacientes mayores de 15 años. 51.350 pacientes presentaron un solo episodio de IAM y 9.301 más de uno (correspondiendo a 20.413 hospitalizaciones). Se excluyeron 291 hospitalizaciones por falta de fecha de egreso, 5 por falta de fecha de ingreso, 6 sin información de edad, 38 por registrar una estadía mayor a 365 días, 5 ocurridos en menores de 15 años y 1.094 sin información de previsión. La muestra final corresponde a 59.557 pacientes.

\section{Análisis estadístico}

Los análisis se estratificaron por sexo. Se calcularon medidas de tendencia central y dispersión y frecuencias absolutas y relativas. Se estimó la sobrevida con el método de Kaplan-Meier para los tres grupos de NSE. Se analizó en forma separada: 1) la sobrevida entre $0-28$ días del ingreso hospitalario y 2) la sobrevida entre 29-365 días, para aislar el efecto del tratamiento agudo y de la prevención secundaria en el pronóstico. Se evaluó la tendencia temporal con regresión de Prais-Winsten (PW), método similar a una regresión lineal, empleado cuando las variables son dependientes del tiempo y los errores están correlacionados con las observaciones inmediatamente contiguas. Su coeficiente expresa en este caso el cambio absoluto en la sobrevida por cada año.

Con regresión multivariada de Cox se estimó: 1) el efecto del NSE en la sobrevida, ajustado por edad y año de hospitalización, usando el NSE alto como referencia y 2) el efecto del año de hospitalización en la sobrevida para cada grupo según NSE ajustado por edad, utilizando el año 2002 como referencia. Los análisis estadísticos se realizaron con el software STATA 13.1. Se consideró como estadísticamente significativo un valor $\mathrm{p} \leq 0,05$.

\section{Resultados}

El número de casos anuales se presentan en la Tabla 1. La mediana de edad fue 64 años (p2575: 54-74); 68,9\% eran hombres. Estos eran más jóvenes en comparación a las mujeres $(61,5 \pm$ 13,1 vs $68,5 \pm 13,5$ años, respectivamente) y de mayor NSE: $59 \%$ de los hombres vs $71,5 \%$ de las mujeres pertenecían al NSE bajo $(\mathrm{p}<0,001)$ (Tabla 2). Las regiones con mayor número de hospitalizaciones fueron: Metropolitana $(38,9 \%)$, Valparaíso (13,8\%) y Biobío (13,6\%). La mayoría de los pacientes FONASA A/B fueron atendidos en hospitales públicos, mientras que los de ISAPREs u otro seguro, en hospitales privados (Tabla 3 ).

\section{Análisis de sobrevida y tendencia}

En las Figuras 1 y 2 se muestra la sobrevida cruda para cada grupo de NSE para los años 20022011. A pesar que entre 0-28 días la sobrevida fue significativamente menor en el grupo de NSE bajo en comparación al NSE alto, el incremento fue significativo en ambos grupos: en mujeres aumentó de $75 \%$ a $84 \%$ y en hombres de $85 \%$ a $90 \%$. El análisis de tendencia mostró mayor incremento en la sobrevida en los pacientes de NSE bajo, principalmente en mujeres: coef PW 1,12: $0,84-1,41$ en mujeres y $0,58: 0,31-0,86$ en hombres entre 0-28 días, lo que indica que por cada año la sobrevida aumentó 1,12 puntos porcentuales en mujeres y 0,58 en hombres. En los pacientes de NSE alto también se registró un aumento en 
Tabla 1. Hospitalizaciones por infarto agudo del miocario (primer episodio) según nivel socioeconómico, Chile 2002-2011

\begin{tabular}{|ccccccccc|}
\hline $\begin{array}{c}\text { Año de } \\
\text { IAM }\end{array}$ & $\begin{array}{c}\text { Total } \\
\mathbf{n}\end{array}$ & $\mathbf{n}$ & $\mathbf{2}$ & \multicolumn{2}{c}{ NSE medio } & \multicolumn{2}{c|}{ NSE alto } \\
\hline 2002 & 4.838 & 2.934 & 60,6 & 1.178 & 24,3 & \multicolumn{1}{c|}{$\mathbf{n}$} & 726 & 15,0 \\
\hline 2003 & 4.939 & 3.137 & 63,5 & 747 & 15,1 & 1.055 & 21,4 \\
\hline 2004 & 5.160 & 3.367 & 65,3 & 851 & 16,5 & 942 & 18,3 \\
\hline 2005 & 5.490 & 3.447 & 62,8 & 893 & 16,3 & 1.150 & 20,9 \\
\hline 2006 & 5.710 & 3.621 & 63,4 & 1.055 & 18,5 & 1.034 & 18,1 \\
\hline 2007 & 6.240 & 3.972 & 63,7 & 1.168 & 18,7 & 1.100 & 17,6 \\
\hline 2008 & 6.360 & 4.081 & 64,2 & 1.214 & 19,1 & 1.065 & 16,7 \\
\hline 2009 & 6.334 & 3.870 & 61,1 & 1.312 & 20,7 & 1.152 & 18,2 \\
\hline 2010 & 7.139 & 4.464 & 62,5 & 1.448 & 20,3 & 1.227 & 17,2 \\
\hline 2011 & 7.347 & 4.550 & 61,9 & 1.561 & 21,2 & 1.236 & 16,8 \\
\hline Total & 59.557 & 37.443 & 62,9 & 11.427 & 19,2 & 10.687 & 17,9 \\
\hline
\end{tabular}

Tabla 2. Características socio-demográficas y distribución de los pacientes hospitalizados por infarto agudo del miocardio, Chile 2002-2011

\begin{tabular}{|c|c|c|c|}
\hline & $\begin{aligned} & \text { Hombres } \\
&(n=41.009 ; 68,9 \%)\end{aligned}$ & $\begin{array}{c}\text { Mujeres } \\
\text { ( } n=18.548 ; 31,1 \%)\end{array}$ & $\begin{array}{c}\text { Total } \\
(n=59.557)\end{array}$ \\
\hline Edad (p50, 25-75), años & $61(52-71)$ & 70 (59-79) & $64(54-74)$ \\
\hline $\begin{array}{c}\text { Previsión: } n(\%) \\
\text { Fonasa A/B } \\
\text { Fonasa C/D } \\
\text { Isapre/ otros }\end{array}$ & $\begin{array}{r}24.179(59,0) \\
8.785(21,4) \\
8.045(19,6)\end{array}$ & $\begin{array}{r}13.264(71,5) \\
2.642(14,2) \\
2.642(14,2)\end{array}$ & $\begin{array}{l}37.443(62,9) \\
11.427(19,2) \\
10.687(17,9)\end{array}$ \\
\hline $\begin{array}{l}\text { Región: n (\%) } \\
\text { I Región de Tarapacá } \\
\text { II Región de Antofagasta } \\
\text { III Región de Atacama } \\
\text { IV Región de Coquimbo } \\
\text { V Región de Valparaíso } \\
\text { VI Región de O`Higgins } \\
\text { VII Región del Maule } \\
\text { VIII Región del Biobío } \\
\text { IX Región de la Araucanía } \\
\text { X Región de los Lagos } \\
\text { XI Región de Aysén } \\
\text { XII Región de Magallanes } \\
\text { Región Metropolitana }\end{array}$ & $\begin{array}{rr}963 & (2,4) \\
1.927 & (4,7) \\
644 & (1,6) \\
1.693 & (4,1) \\
5.673 & (13,8) \\
1.706(4,2) \\
1.850(4,5) \\
5.330(13,0) \\
1.983(4,8) \\
2.703(6,6) \\
158(0,4) \\
444(1,1) \\
15.935(38,9)\end{array}$ & $\begin{array}{rr}386 & (2,1) \\
642 & (3,5) \\
209 & (1,1) \\
742 & (4,0) \\
2.799 & (15,1) \\
763 & (4,1) \\
828 & (4,5) \\
2.715 & (14,6) \\
925 & (5,0) \\
1.175 & (6,3) \\
44 & (0,2) \\
143 & (0,8) \\
7.177 & (38,7)\end{array}$ & $\begin{array}{rr}1.349 & (2,3) \\
2.569 & (4,3) \\
853 & (1,4) \\
2.435 & (4,1) \\
8.472 & (14,2) \\
2.469 & (4,2) \\
2.678 & (4,5) \\
8.045 & (13,5) \\
2.908 & (4,9) \\
3.878 & (6,5) \\
202 & (0,3) \\
587 & (1,0) \\
23.112 & (38,8)\end{array}$ \\
\hline
\end{tabular}

Tabla 3. Nivel socioeconómico (NSE) y tipo de hospital en pacientes hospitalizados por infarto agudo del miocardio, Chile 2002-2011

\begin{tabular}{|c|c|c|c|c|c|c|}
\hline \multirow[t]{2}{*}{ Nivel socioeconómico } & \multicolumn{2}{|c|}{$\begin{array}{l}\text { Hospital público } \\
(n=49.558)\end{array}$} & \multicolumn{2}{|c|}{$\begin{array}{l}\text { Hospital privado } \\
(n=9.999)\end{array}$} & \multicolumn{2}{|c|}{$\begin{array}{c}\text { Total } \\
(\mathrm{n}=\mathbf{5 9 . 5 5 7 )}\end{array}$} \\
\hline & n & $\%$ & n & $\%$ & n & $\%$ \\
\hline NSE bajo & 36.863 & 74,38 & 580 & 5,80 & 37.443 & 62,87 \\
\hline NSE medio & 9.690 & 19,55 & 1.737 & 17,37 & 11.427 & 19,19 \\
\hline NSE alto & 3.005 & 6,06 & 7.682 & 76,83 & 10.687 & 17,94 \\
\hline
\end{tabular}




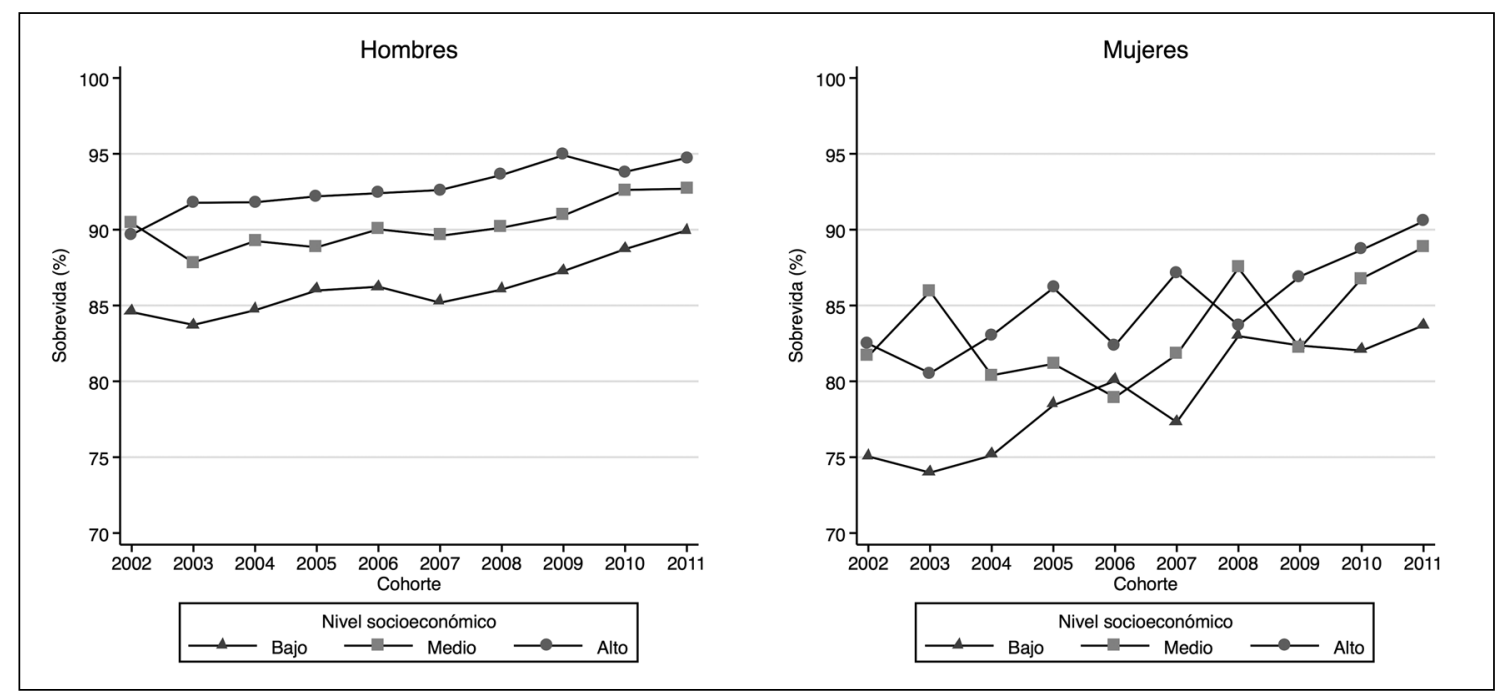

Figura 1. Sobrevida post primer infarto agudo al miocardio entre 0 y 28 días por causas cardiovasculares, según sexo y nivel socioeconómico. Chile 2002-2012.

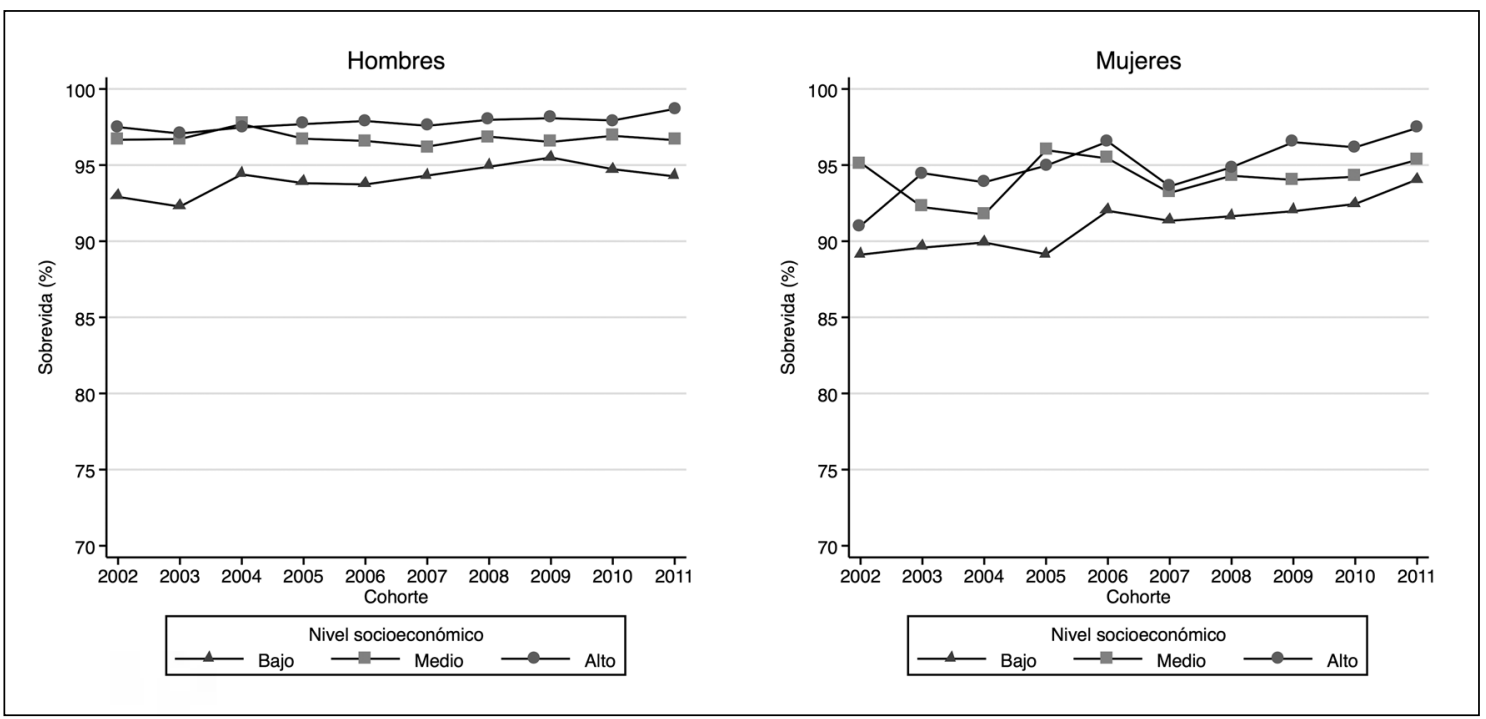

Figura 2. Sobrevida post primer infarto agudo al miocardio entre 29 y 365 días por causas cardiovasculares, según sexo y nivel socioeconómico. Chile 2002-2012.

la sobrevida en ambos sexos. Entre 29-365 días el incremento en la sobrevida fue menor, pero también significativo para los grupos de bajo y alto NSE (Figura 2), siendo mayor en las mujeres: coef PW 0,48: 0,37-0,60 mujeres y 0,24: 0,09-0,39 hombres (Tabla 4).

$\mathrm{El}$ análisis multivariado mostró que el riesgo de muerte cardiovascular durante los primeros 28 días en hombres de NSE bajo fue 1,67 veces mayor respecto a los de NSE alto. En aquellos que sobrevivieron al día 28 , este riesgo aumentó a 2,3 veces. De forma similar, las mujeres de los grupos bajo y medio presentaron mayor riesgo de muerte comparadas con el estrato alto, tanto en el seguimiento precoz como alejado, pero estos fueron menores que en hombres (Tabla 5).

Para identificar el año de ocurrencia en el cual mejoró la sobrevida, se compararon los hazard 
Tabla 4. Tendencia temporal de sobrevida en pacientes hospitalizados post infarto agudo al miocardio por causas cardiovasculares (coeficientes de regresión Prais -Winsten) en hombres y mujeres según nivel socioeconómico, Chile 2002-2011

\begin{tabular}{|c|c|c|c|c|}
\hline & & Coeficie & IC95 \%) & \\
\hline & 0-28 días & $\mathbf{p}$ & 29-365 días & $\mathbf{p}$ \\
\hline Hombre & & & & \\
\hline NSE bajo & $0,58 \quad(0,31-0,86)$ & 0,001 & $0,24 \quad(0,09-0,39)$ & 0,007 \\
\hline NSE medio & $0,41 \quad(0,17-0,64)$ & 0,004 & $-0,03(-0,12-0,06)$ & 0,47 \\
\hline NSE alto & $0,47 \quad(0,35-0,59)$ & $<0,001$ & $0,12 \quad(0,09-0,15)$ & $<0,001$ \\
\hline Mujer & & & & \\
\hline NSE bajo & $1,12(0,84-1,41)$ & $<0,001$ & $0,48 \quad(0,37-0,60)$ & $<0,001$ \\
\hline NSE medio & $0,61(-0,15-1,37)$ & 0,10 & $0,12(-0,20-0,46)$ & 0,40 \\
\hline NSE alto & $0,88(0,60-1,17)$ & $<0,001$ & $0,45(0,20-0,71)$ & 0,003 \\
\hline
\end{tabular}

Tabla 5. Hazards ratios ajustados (IC $95 \%$ ) para muerte cardiovascular en pacientes hospitalizados por infarto agudo del miocardio, según sexo y nivel socioeconómico, Chile 2002-2011

\begin{tabular}{|lccc|}
\hline & n & \multicolumn{2}{c|}{ Hazard ratios* (IC 95\%) } \\
29-365 días \\
Hombre & & $\mathbf{0 - 2 8}$ días & \\
NSE bajo & 24.179 & $1,67(1,53-1,83)$ & $2,30(1,75-2,71)$ \\
NSE medio & 8.785 & $1,42(1,26-1,57)$ & $1,60(1,31-1,95)$ \\
NSE alto & 8.045 & 1 & 1 \\
Mujer & & & $1,90(1,56-2,32)$ \\
NSE bajo & 13.264 & $1,49(1,34-1,66)$ & $1,43(1,10-1,85)$ \\
NSE medio & 2.642 & $1,29(1,12-1,48)$ & 1 \\
NSE alto & 2.642 & 1 & 1 \\
\hline
\end{tabular}

*ajustados por edad y año calendario de hospitalización.

ratios (HRs) de cada cohorte año calendario con el año $2002(\mathrm{HR}=1,00)$. A partir de 2009 se observó menores riesgos de muerte entre 0 -28 días para el NSE bajo, disminución que no resultó significativa para el NSE alto. En las mujeres, desde 2008, el grupo de NSE bajo presentó menor mortalidad cardiovascular en comparación al año 2002 (Figura 3). Dicha mejoría fue significativa también para quienes sobrevivieron al día 28, en hombres y mujeres, a partir de 2008 y 2010, respectivamente (Figura 4).

\section{Discusión}

Este estudio muestra un aumento en la sobrevida precoz y alejada post IAM en pacientes hospitalizados entre 2002 y 2011; sin embargo, se mantuvo una distribución inequitativa de la mortalidad, en desmedro de los grupos de menor nivel socioeconómico, en ambos sexos. El resultado más destacable es el marcado aumento en la sobrevida precoz (0-28 días) en el grupo de bajo NSE, resultado que no se observó en el nivel alto. Esta reducción podría explicarse, en parte, por el mejor acceso a diagnóstico precoz y tratamientos de reperfusión y revascularización, al disponerse de mayores recursos para el sistema público de salud $^{25,28}$. En la fase tardía el incremento en la sobrevida fue menor, lo que podría deberse a la falta de centros de rehabilitación cardiaca, la ausencia de una estrategia consolidada de prevención secundaria en el sector público, y a la baja indicación de estos programas en el sector privado ${ }^{29,30}$. La evidencia sobre el deficiente acceso a tratamientos posterior a un IAM se relaciona con peor pronóstico en el largo plazo ${ }^{31}$; también la dificultad para 


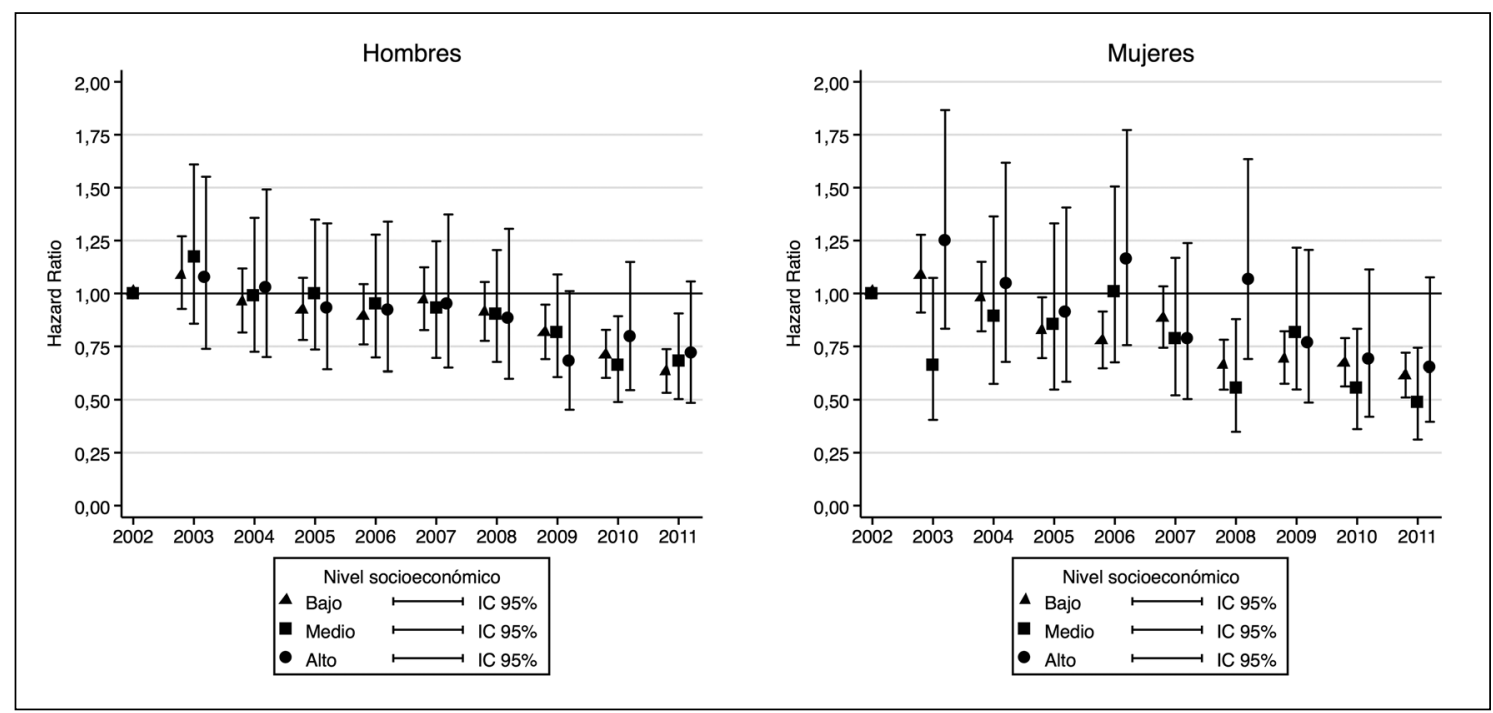

Figura 3. Evolución de los hazard ratios de fallecer por causa cardiovascular en los primeros 28 días posterior a un primer infarto agudo al miocardio hospitalizado, según sexo y nivel socioeconómico. Chile 2002-2012.

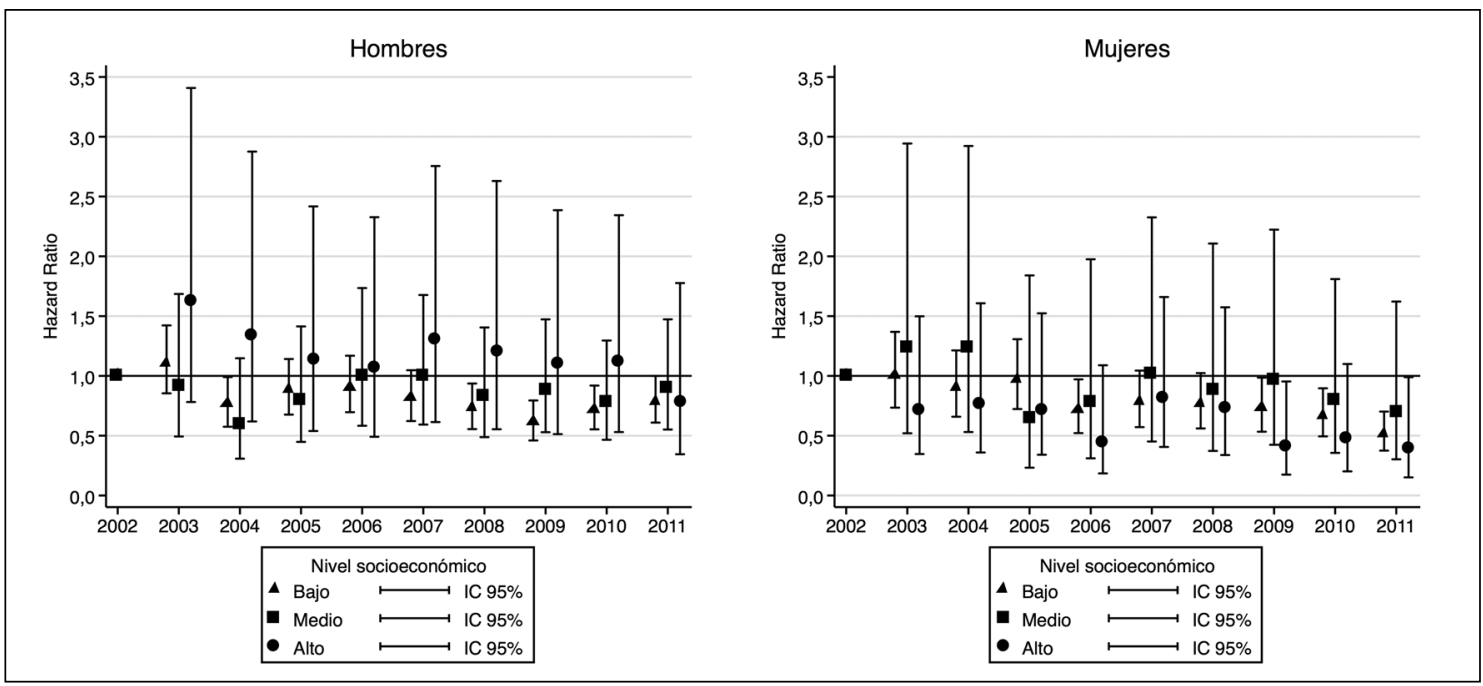

Figura 4. Evolución de los hazard ratios de fallecer por causa cardiovascular entre el día 29 a 365 posterior a un primer infarto agudo al miocardio hospitalizado, según sexo y nivel socioeconómico. Chile 2002-2012.

adoptar estilos de vida saludables que es mayor en pacientes de bajo NSE ${ }^{32}$. En cambio, la participación en programas de prevención secundaria mejora el pronóstico ${ }^{33,34}$.

La menor sobrevida en las mujeres en las cifras crudas puede explicarse parcialmente por la diferencia de edad, dado que eran en promedio 7 años mayores que los hombres. Varios estudios, incluida una revisión sistemática, muestran que posterior al ajuste por edad la diferencia de riesgo de muerte entre hombres y mujeres disminuye significativamente ${ }^{35-37}$. La primera evaluación realizada en Chile después del GES mostró que en las mujeres la reducción de la mortalidad intrahospitalaria fue mayor que en hombres, relacionada con el incremento en la terapia farmacológica, procedimientos de reperfusión y de revascularización en ellas ${ }^{38}$. 
La principal fortaleza del estudio es que cubre una década de hospitalizaciones por IAM, con representatividad nacional, empleando datos poblacionales. Además, se presentan análisis separados por sexo y para sobrevida precoz y alejada, con el fin de entregar información respecto de las estrategias terapéuticas implementadas durante el período que impactan en el corto y largo plazo.

Entre las limitaciones, en primer lugar se destaca que la fuente de información no permite identificar los infartos con y sin SDST; estos últimos pueden haber aumentado dado el incremento progresivo de empleo de troponinas en el sector público, lo que podría influir en la menor mortalidad en los últimos años en este sector. Dado que hay perdida de observaciones, principalmente en los años al inicio del período estudiado, no es posible cuantificar el cambio real en las hospitalizaciones anuales. En segundo lugar, se usó el seguro de salud como medida de posición socioeconómica, al no disponerse de otros indicadores más complejos -por ejemplo que permiten capturar la exposición a condiciones desfavorables durante el curso de la vida-, que podrían explicar de mejor manera dicha asociación ${ }^{39-41}$. Sin embargo, la diferenciación de los tramos del seguro público, basada en el ingreso de los afiliados, permite establecer una gradiente socioeconómica para este análisis. En tercer lugar la muestra analizada sólo representa a pacientes que alcanzan a ser hospitalizados por IAM, por tanto no se entrega evidencia sobre la atención extrahospitalaria, ni los resultados representan a toda la población afectada. Finalmente, no se dispone de información clínica ni terapéutica que permita realizar ajustes en el análisis por estas variables.

La revisión sistemática de Schröder releva la existencia de inequidades socioeconómicas en pacientes coronarios, lo cual no se ha resuelto necesariamente por la implementación de cobertura universal ${ }^{42}$. En Chile, en los últimos años ha mejorado el acceso de los pacientes del sector público a procedimientos angiográficos diagnósticos y de reperfusión ${ }^{28}$, así como a terapias efectivas en la fase aguda y alejada, lo que se traduce en la mejoría de la sobrevida en pacientes de menor nivel socioeconómico.

La enfermedad coronaria está priorizada en los Objetivos Sanitarios para la Década 2011-2020, cuya meta específica es aumentar la sobrevida a un año post infarto ${ }^{43}$. Estos resultados serán de utilidad para la evaluación de las políticas públicas implementadas, reconociendo que se requiere la participación de otros sectores para abordar de manera efectiva este problema.

En conclusión, a pesar del aumento registrado en la sobrevida en ambos sexos y todos los grupos según nivel socioeconómico, aun se observan inequidades en la mortalidad por IAM de los pacientes hospitalizados durante la última década en Chile. El análisis de tendencia temporal muestra un aumento mayor de la sobrevida en pacientes de bajo nivel socioeconómico, principalmente en mujeres.

\section{Referencias}

1. Smolina K, Wright FL, Rayner M, Goldacre MJ. Determinants of the decline in mortality from acute myocardial infarction in England between 2002 and 2010: linked national database study. BMJ 2012; 344: d8059.

2. Ford ES, Ajani UA, Croft JB, Critchley JA, Labarthe DR, Kottke TE, et al. Explaining the decrease in U.S. deaths from coronary disease, 1980-2000. N Engl J Med 2007; 356 (23): 2388-98.

3. Ministerio de Salud de Chile. Los Objetivos Sanitarios para la Década 2000-2010 Evaluación de final del período, Grado de cumplimiento de los objetivos de Impacto. 2010 [Consultado el 9 de diciembre de 2010]; Available from: http://epi.minsal.cl/epi/html/sdesalud/OS/EvaluacionObjetivosSanitarios2000-2010.pdf.

4. Nazzal C, Alonso FT. [Incidence and case fatality due to acute myocardial infarction in Chile in the period 2001-2007]. Rev Med Chile 2011; 139 (10): 1253-60.

5. Avendaño M, Kunst AE, Huisman M, Lenthe FV, Bopp $\mathrm{M}$, Regidor $\mathrm{E}$, et al. Socioeconomic status and ischaemic heart disease mortality in 10 western European populations during the 1990s. Heart 2006; 92 (4): 461-7.

6. Cardona D, Acosta LD, Bertone CL. [Inequities in health among Latin American and Caribbean countries (20052010)]. Gac Sanit 2013; 27 (4): 292-7.

7. Gerber Y, Weston SA, Killian JM, Therneau TM, Jacobsen SJ, Roger VL. Neighborhood income and individual education: effect on survival after myocardial infarction. Mayo Clin Proc. 2008; 83 (6): 663-9.

8. Nazzal C, Corbalán R, Díaz C, Sepúlveda P, Schacht E. [Effect of educational level on the prognosis of acute myocardial infarction]. Rev Med Chile 2015; 143 (7): 825-33.

9. Rose G, Marmot MG. Social class and coronary heart disease. Br Heart J 1981; 45 (1): 13-9. 
10. Kaplan GA, Keil JE. Socioeconomic factors and cardiovascular disease: a review of the literature. Circulation 1993; 88 (4 Pt 1): 1973-98.

11. Emberson JR, Whincup PH, Morris RW, Walker M. Social class differences in coronary heart disease in middle-aged British men: implications for prevention. Int J Epidemiol 2004; 33 (2): 289-96.

12. Salomaa V, Miettinen $H$, Niemela $M$, Ketonen $M$, Mahonen M, Immonen-Raiha $\mathrm{P}$, et al. Relation of socioeconomic position to the case fatality, prognosis and treatment of myocardial infarction events; the FINMONICA MI Register Study. J Epidemiol Community Health 2001; 55 (7): 475-82.

13. Yusuf S, Islam S, Chow CK, Rangarajan S, Dagenais G, Diaz R, et al. Use of secondary prevention drugs for cardiovascular disease in the community in high-income, middle-income, and low-income countries (the PURE Study): a prospective epidemiological survey. Lancet 2011; 378 (9798): 1231-43.

14. Nazzal CCR, Frenz P, Sepúlveda P, Prieto J. Differences in treatment and in-hospital mortality of acute myocardial infarction patients in public and private hospitals in Chile before and after healthcare guarantees (abstract). European Society of Cardiology Congress; Amsterdam 2013.

15. Kivimaki M, Lawlor DA, Davey Smith G, Kouvonen A, Virtanen M, Elovainio M, et al. Socioeconomic position, co-occurrence of behavior-related risk factors, and coronary heart disease: the Finnish Public Sector study. Am J Public Health 2007; 97 (5): 874-9.

16. Ministerio de Salud GdC. Encuesta Nacional de Salud. Chile 2009-2010. 2011 [consultado el 4 de marzo de 2014]; Available from: http://www.redsalud.gov.cl/ portal/docs/page/minsalcl/g_home/submenu_portada_2011/ens2010.pdf.

17. Sundquist J, Malmstrom M, Johansson SE. Cardiovascular risk factors and the neighbourhood environment: a multilevel analysis. Int J Epidemiol 1999; 28 (5): 841-5.

18. Diez-Roux AV, Nieto FJ, Muntaner C, Tyroler HA, Comstock GW, Shahar E, et al. Neighborhood environments and coronary heart disease: a multilevel analysis. Am J Epidemiol 1997; 146 (1): 48-63.

19. Peters A, Dockery DW, Muller JE, Mittleman MA. Increased particulate air pollution and the triggering of myocardial infarction. Circulation 2001; 103 (23): 28105.

20. Tonne C, Halonen JI, Beevers SD, Dajnak D, Gulliver J, Kelly FJ, et al. Long-term traffic air and noise pollution in relation to mortality and hospital readmission among myocardial infarction survivors. Int J Hyg Environ Health 2016; 219 (1): 72-8.
21. Birch M. Implementing equity: the Commission on Social Determinants of Health. Bull World Health Organ 2009; 87 (1): 3.

22. PAHO. Plan of Action for the Prevention and Control of Noncomunicable Diseases in the Americas 2013-2019. Washington (D.C.): Pan American Health Organization; 2014. 2014.

23. Di Cesare M, Khang YH, Asaria P, Blakely T, Cowan MJ, Farzadfar F, et al. Inequalities in non-communicable diseases and effective responses. Lancet 2013; 381 (9866): 585-97.

24. Salinas J, Cancino A, Pezoa S, Salamanca F, Soto M. [The Vida Chile program: results and challenges with health promotion policy in Chile, 1998-2006]. Rev Panam Salud Publica 2007; 21 (2-3): 136-44.

25. Frenz P, Delgado I, Kaufman JS, Harper S. Achieving effective universal health coverage with equity: evidence from Chile. Health Policy Plan. 2013 Aug 5.

26. FONASA. Clasificación Asegurados según Tramos [citado el 7 de julio de 2017]; Available from: https:// fonasaweb.fonasa.cl/portal_fonasa/site/artic/20140621/ pags/20140621192134.html

27. Ministerio de Desarrollo Social-Gobierno de Chile. Encuesta de Caracterización Socioeconómica Nacional, CASEN 2011. 2012.

28. Nazzal C, Frenz P, Alonso FT, Lanas F. Effective universal health coverage and improved 1-year survival after acute myocardial infarction: the Chilean experience. Health Policy and Planning. 2015.

29. Santíbañez C, Pérez-Terzic C, López-Jiménez F, Cortés-Bergoderi M, Araya MV, Burdiat G. [Current status of cardiac rehabilitation in Chile]. Rev Med Chile 2012; 140 (5): 561-8.

30. Nazzal C, Lanas F, Garmendia ML, Bugueno C, Mercadal E, Garces E, et al. [Universal health coverage and accomplishment of secondary prevention goals among patients with acute myocardial infarction]. Rev Med Chile 2013; 141 (8): 977-86.

31. Rahimi AR, Spertus JA, Reid KJ, Bernheim SM, Krumholz HM. Financial barriers to health care and outcomes after acute myocardial infarction. JAMA 2007; 297 (10): 1063-72.

32. Gaalema DE, Elliott RJ, Morford ZH, Higgins ST, Ades PA. Effect of Socioeconomic Status on Propensity to Change Risk Behaviors Following Myocardial Infarction: Implications for Healthy Lifestyle Medicine. Prog Cardiovasc Dis. 2017 Jan 05.

33. Perk J, De Backer G, Gohlke H, Graham I, Reiner Z, Verschuren WM, et al. European Guidelines on cardiovascular disease prevention in clinical practice (version 2012): The Fifth Joint Task Force of the European Socie- 
ty of Cardiology and Other Societies on Cardiovascular Disease Prevention in Clinical Practice (constituted by representatives of nine societies and by invited experts). Atherosclerosis 2012; 223 (1): 1-68.

34. Smith SC Jr, Allen J, Blair SN, Bonow RO, Brass LM, Fonarow GC, et al. AHA/ACC guidelines for secondary prevention for patients with coronary and other atherosclerotic vascular disease: 2006 update: endorsed by the National Heart, Lung, and Blood Institute. Circulation 2006; 113 (19): 2363-72.

35. Corrada E, Ferrante G, Mazzali C, Barbieri P, Merlino L, Merlini $\mathrm{P}$, et al. Eleven-year trends in gender differences of treatments and mortality in ST-elevation acute myocardial infarction in northern Italy, 2000 to 2010 . Am J Cardiol 2014; 114 (3): 336-41.

36. Leurent G, Garlantezec R, Auffret V, Hacot JP, Coudert I, Filippi E, et al. Gender differences in presentation, management and inhospital outcome in patients with ST-segment elevation myocardial infarction: data from 5000 patients included in the ORBI prospective French regional registry. Arch Cardiovasc Dis 2014; 107 (5): 291-8.

37. Bucholz EM, Butala NM, Rathore SS, Dreyer RP, Lansky AJ, Krumholz HM. Sex differences in long-term mortality after myocardial infarction: a systematic review. Circulation 2014; 130 (9): 757-67.

38. Nazzal NC, Campos TP, Corbalán HR, Lanas ZF, Bar- tolucci JJ, Sanhueza CP, et al. [The impact of Chilean health reform in the management and mortality of ST elevation myocardial infarction (STEMI) in Chilean hospitals]. Rev Med Chile 2008; 136 (10): 1231-9.

39. Galobardes B, Shaw M, Lawlor DA, Lynch JW, Davey Smith G. Indicators of socioeconomic position (part 1). J Epidemiol Community Health 2006; 60 (1): 7-12.

40. Galobardes B, Shaw M, Lawlor DA, Lynch JW, Davey Smith G. Indicators of socioeconomic position (part 2). J Epidemiol Community Health 2006; 60 (2): 95101.

41. Ljung R, Hallqvist J. Accumulation of adverse socioeconomic position over the entire life course and the risk of myocardial infarction among men and women: results from the Stockholm Heart Epidemiology Program (SHEEP). J Epidemiol Community Health 2006; 60 (12): 1080-4.

42. Schroder SL, Richter M, Schroder J, Frantz S, Fink A. Socioeconomic inequalities in access to treatment for coronary heart disease: A systematic review. Int J Cardiol 2016; 219: 70-8.

43. Ministerio de Salud Gobierno de Chile. Estrategia Nacional de Salud Para el cumplimiento de los Objetivos Sanitarios de la Década 2011-2020. 2011 [citado el 10 de noviembre de 2013]; Available from: http://web.minsal.cl/portal/url/item/c4034eddbc96ca6de0400101640159b8.pdf. 